\title{
Diversification of 4'-Methylated Nucleosides by Nucleoside Phosphorylases
}

Felix Kaspar, ${ }^{*[1,2]}$ Margarita Seeger, ${ }^{[3]}$ Sarah Westarp,${ }^{[1,2]}$ Christoph Köllmann, ${ }^{[4]}$ Anna P. Lehmann, ${ }^{[4]}$ Patrick Pausch, ${ }^{[5], \#}$ Sebastian Kemper, ${ }^{[6]}$ Peter Neubauer, ${ }^{[1]}$ Gert Bange, ${ }^{[5]}$ Anett Schallmey, ${ }^{[3]}$ Daniel B. Werz, ${ }^{[4]}$ Anke Kurreck ${ }^{[1,2]}$

${ }^{1}$ Chair of Bioprocess Engineering, Institute of Biotechnology, Faculty III Process Sciences, Technische Universität Berlin, Ackerstraße 76, D-13355 Berlin, Germany.

${ }^{2}$ BioNukleo GmbH, Ackerstraße 76, D-13355 Berlin, Germany.

${ }^{3}$ Technische Universität Braunschweig, Institute for Biochemistry, Biotechnology and Bioinformatics, Spielmannstraße 7, 38106, Braunschweig, Germany.

${ }^{4}$ Technische Universität Braunschweig, Institute of Organic Chemistry, Hagenring 30, 38106, Braunschweig, Germany.

${ }^{5}$ Center for Synthetic Microbiology (SYNMIKRO) \& Department of Chemistry, Philipps-University Marburg, HansMeerwein-Strasse 6, C07, 35043 Marburg, Germany.

${ }^{6}$ Institute for Chemistry, Technische Universität Berlin, Straße des 17. Juni 135, 10623 Berlin, Germany.

\# Present address: Innovative Genomics Institute, University of California, Berkeley, 2151 Berkeley Way, Berkeley, CA 94720, USA

The growing demand for 4'-modified nucleoside analogs in medicinal and biological chemistry is contrasted by the challenging synthetic access to these molecules and the lack of efficient diversification strategies. Herein, we report the development of a biocatalytic diversification approach based on nucleoside phosphorylases, which allows the straightforward installation of a variety of pyrimidine and purine nucleobases on a 4'-alkylated sugar scaffold. Following the identification of a suitable biocatalyst as well as its characterization with kinetic experiments and docking studies, we systematically explored the equilibrium thermodynamics of this reaction system to enable rational yield prediction in transglycosylation reactions via principles of thermodynamic control.

Nucleosides are central biomolecules that play key roles in a variety of cellular processes by serving as enzymatic cofactors, building blocks of DNA and RNA and energy transport systems. As such, modified nucleosides mimicking their natural counterparts have a long history in medicinal and biological chemistry. ${ }^{[1-4]}$ Today, modified nucleosides are indispensable pharmaceuticals for the treatment of various types of cancer and viral infections and further represent important tools in chemical biology for a spectrum of imaging applications. ${ }^{[5,6]}$ Despite the great demand for these molecules, the synthesis of nucleosides is still regarded as challenging and inefficient. ${ }^{[7]}$ While nucleosides with ribosyl or 2'-desoxyribosyl moieties can be accessed from naturally occurring nucleosides or carbohydrates, ${ }^{[7-10]}$ the preparation of sugar-modified nucleosides typically suffers from lengthy reaction sequences and low total yields. ${ }^{[1-18]}$ Furthermore, a heavy reliance on protecting groups entails low overall efficiencies $^{[7]}$ and several sugar modifications at the $2^{\prime}$ or $4^{\prime}$ positions are known to limit diastereoselectivity in glycosylation approaches, ${ }^{[19,20]}$ severely complicating the synthetic access to many target compounds. More importantly, established routes typically exhibit a lack of divergence as they tend to be specific to one nucleoside. As such, the introduction of desired substitutions at the nucleobase often requires complete or partial re-synthesis of the target molecule since a general strategy for the efficient diversification of modified nucleosides has not been reported to date (Scheme 1, top). With the advent of scalable routes for the de novo synthesis of selected 4'-modified nucleoside analogs, as reported recently by Britton, ${ }^{[21]}$ such a diversification strategy would readily provide access to a variety of sought-after nucleosides.

We envisioned that nucleoside phosphorylases could provide a biocatalytic platform for late-stage diversification of 4'-modified nucleosides. These enzymes catalyze the reversible phosphorolysis of nucleosides to the corresponding nucleobases and pentose-1-phosphates via an $\mathrm{S}_{\mathrm{N}}$ 2-like mechanism. ${ }^{[22,23]}$ The reaction sequence involving phosphorolysis of one nucleoside and in situ reverse phosphorolysis to the target nucleoside is generally known as a transglycosylation, and effectively transfers the sugar moiety from one nucleobase to another. ${ }^{[24]}$ While this reactivity is well-established for ribosyl and 2'desoxyribosyl nucleosides ${ }^{[9]}$ and a few $2^{\prime}$-modified nucleosides (Scheme 1, center), there are no examples in the literature of the enzymatic synthesis of 4'-modified nucleosides, except for Merck's recent report of a 5-step enzymatic cascade for the synthesis of the 4 '-alkynylated nucleoside drug Islatravir. ${ }^{[25]}$ Therefore, the feasibility of transglycosylation reactions with 4 '-modified nucleosides as well as the thermodynamics of such a cascade process are notably underexplored. Herein, we address this gap by reporting on the phosphorolysis and transglycosylation of the simplest 4'-alkylated pyrimidine nucleoside, 4'methyluridine (1a). Following the identification of a suitable biocatalyst, and a characterization of its reactivity with kinetic experiments and docking studies, we explored the thermodynamics of the phosphorolysis of $\mathbf{1 a}$ and leveraged this information in transglycosylation experiments to access a range of $4^{\prime}$-methylated pyrimidine and purine nucleosides.

In the absence of obvious pyrimidine nucleoside phosphorylase (PyNP) candidates for the phosphorolysis of 1a, we began our investigation by screening a small panel of PyNPs with known broad substrate spectra. To our surprise, only the PyNP from Thermus thermophilus $(T t \text { PyNP })^{[26,27]}$ showed measurable conversion of 1a under screening conditions (Figure S1). Other broad-spectrum PyNPs, such as those from Geobacillus thermoglucosidasius (GtPyNP) ${ }^{[28]}$ or Bacillus subtilis, ${ }^{[23]}$ displayed no activity with 1a (Figure 1A). To substantiate the observed conversion of $\mathbf{1 a}$ by $T t \mathrm{PyNP}$, we performed a series of control experiments. Reactions either without 


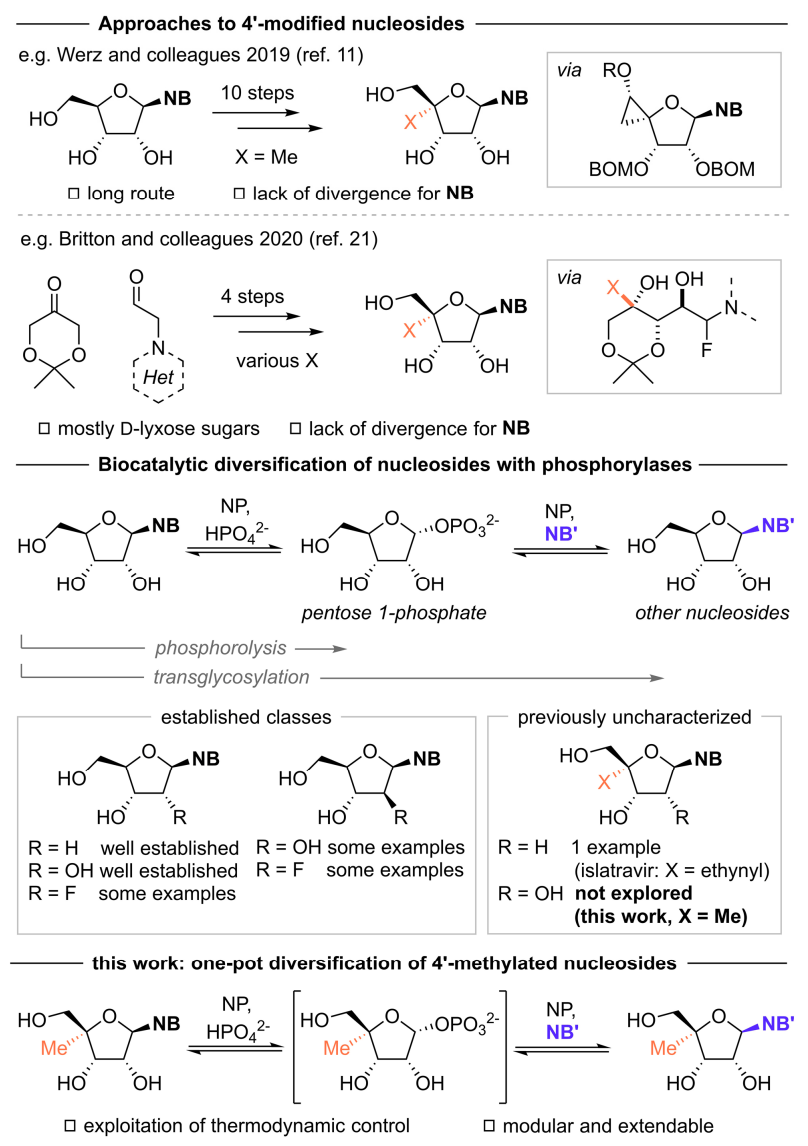

Scheme 1. Synthesis and biocatalytic diversification of nucleosides with modified sugars. $\mathrm{NB}=$ Nucleobase, $\mathrm{NP}=$ nucleoside phosphorylase.

phosphate, without enzyme or with denatured enzyme gave no conversion. Similarly, no conversion was observed under reaction conditions outside of the working space of $T t \mathrm{PyNP}$ $\left(\mathrm{pH} 3\right.$ or $\mathrm{pH} 12$, Figure 1A). ${ }^{[26]} \mathrm{NMR}$ analysis of a reaction mixture with TtPyNP and 1a corroborated the proposed reactivity and creation of the pentose-1-phosphate $\mathbf{3}$, as evident from the rise of an additional ${ }^{1} \mathrm{H}$ NMR signal at $5.57 \mathrm{ppm}$ showing a strong H,P-HMQC signal (Figure 1B). Consistent with the native reactivity of PyNPs, inversion at the anomeric position was evident by this signal lacking NOE contacts to the 4'-methyl group of $\mathbf{3}$, while the corresponding anomeric proton in 1a showed clear correlation to the methyl substituent.

Having established the activity of TtPyNP with 1a, we conducted kinetic experiments to provide further insights into this enzymatic transformation. Although TtPyNP is inhibited by pyrimidine nucleobases such as uracil $(\mathbf{2 a}),{ }^{[26]}$ we could observe Michaelis-Menten behavior of the enzyme with 1a (Figure 1C). Interestingly, the apparent MichalisMenten constant $K_{\mathrm{M}}{ }^{\prime}$ of the phosphorolysis of $\mathbf{1 a}\left(K_{\mathrm{M}^{\prime}}=\right.$ $3.37 \mathrm{mM}$ ) indicated that $T t$ PyNP has a much lower affinity for 1a compared to natural nucleosides like uridine or thymidine $\left(K_{\mathrm{M}}<1 \mathrm{mM}\right),{ }^{[27]}$ suggesting that productive binding of the modified substrate 1a might present a challenge due to the increased steric bulk. In addition to a lower affinity for 1a, TtPyNP also displayed a lower rate constant compared to uridine $\left(0.59\right.$ vs $5.05 \mathrm{~s}^{-1}$ for $1 \mathrm{mM}$ substrate at $60{ }^{\circ} \mathrm{C}$ and $\left.\mathrm{pH} 9\right)^{[26]}$ which showed a similar temperature-dependence as indicated by phosphorolysis experiments at different temperatures monitored by UV spectroscopy (Figure 1D). ${ }^{[29,30]}$ Collectively, these results demonstrate that, unlike other nucleoside phosphorylases, $T t$ PyNP selectively converts the 4'-methylated nucleoside 1a to the corresponding sugar phosphate 3, albeit with a lower rate constant and substrate affinity compared to the native substrates.

Next, we performed preliminary in silico docking studies to rationalize why $\mathbf{1 a}$ is only converted by TtPyNP and not by other closely related and highly promiscuous enzymes such as GtPyNP. We hypothesized that conversion of this substrate would primarily be limited by steric hindrance during substrate binding, since i) uridine and 1a only differ by a single methyl group distant from the anomeric position and ii) TtPyNP displays significantly lower affinity for $\mathbf{1 a}$ than for uridine. PyNPs generally exhibit marked flexibility during their catalytic cycle with a transition from an open conformation to a closed state requiring a domain movement of approximately $8 \AA \AA^{[31]}$ Since all first sphere residues in the closed state are highly conserved and identical between the tested PyNPs, we anticipated that initial binding in the open conformation would be a limiting factor, as TtPyNP offers slightly more space than GtPyNP due to a threonine-serine substitution at the back of the active site, as evident from sequence alignments. ${ }^{[28]}$ To examine this hypothesis, we obtained an X-ray crystal structure of GtPyNP at $1.9 \AA$ resolution (see Supporting Information for details; PDB ID 7m7k) and used AutoDock Vina implemented in YASARA to dock uridine and $1 \mathbf{a}$ into the open conformations of this structure and the known Xray crystal structure of $T t$ PyNP (PDB ID 2dsj). ${ }^{[32]}$ Docking of uridine and 1a into $T t$ PyNP yielded structures in good agreement with the native mode of substrate binding via $\mathrm{H}$ bonding to the nucleobase and positioning of the anomeric carbon near the phosphate binding pocket (Figures 2A and 2B). Likewise, uridine could be docked into GtPyNP in a similar position to the cocrystallized substrate (Figures $2 \mathrm{C}$ and S8), where the 4'-position of uridine is located in proximity to Thr84 (Ser83 in TtPyNP). However, we were unable to obtain sensible docking results for 1a with GtPyNP as the increased steric bulk at the 4'-position consistently led to a rotation of the sugar scaffold into an unproductive pose (Figure 2D). This suggested that the subtle space-creating mutation to a serine in TtPyNP might be a key factor for conversion of 1a. Consistent with this conclusion, the slightly more sterically congested TtPyNPS83T mutant significantly lost activity compared to the parent enzyme $\left(k_{\mathrm{obs}}=0.25 \mathrm{~s}^{-1}\right.$ vs $k_{\mathrm{obs}}=0.59 \mathrm{~s}^{-1}$, Table S2), while the reverse substitution in GtPyNP installed a low but measurable level of activity in this enzyme $\left(k_{\mathrm{obs}}=0.02 \mathrm{~s}^{-1}\right.$ for GtPyNP-T84S). Moreover, all other enzymes we screened initially, and which were inactive with 1a, also possess a threonine at this position, which likely impedes their ability to bind this substrate productively. Although such subtle but crucial space-creating mutations are rare, there is precedent from other enzymes in the literature. ${ }^{[34]}$ Together, these results indicate that sufficient space in the open conformation of PyNPs is a prerequisite for conversion of sterically more demanding substrates such as 1a. Clearly, there are other factors influencing the rate constant of this 

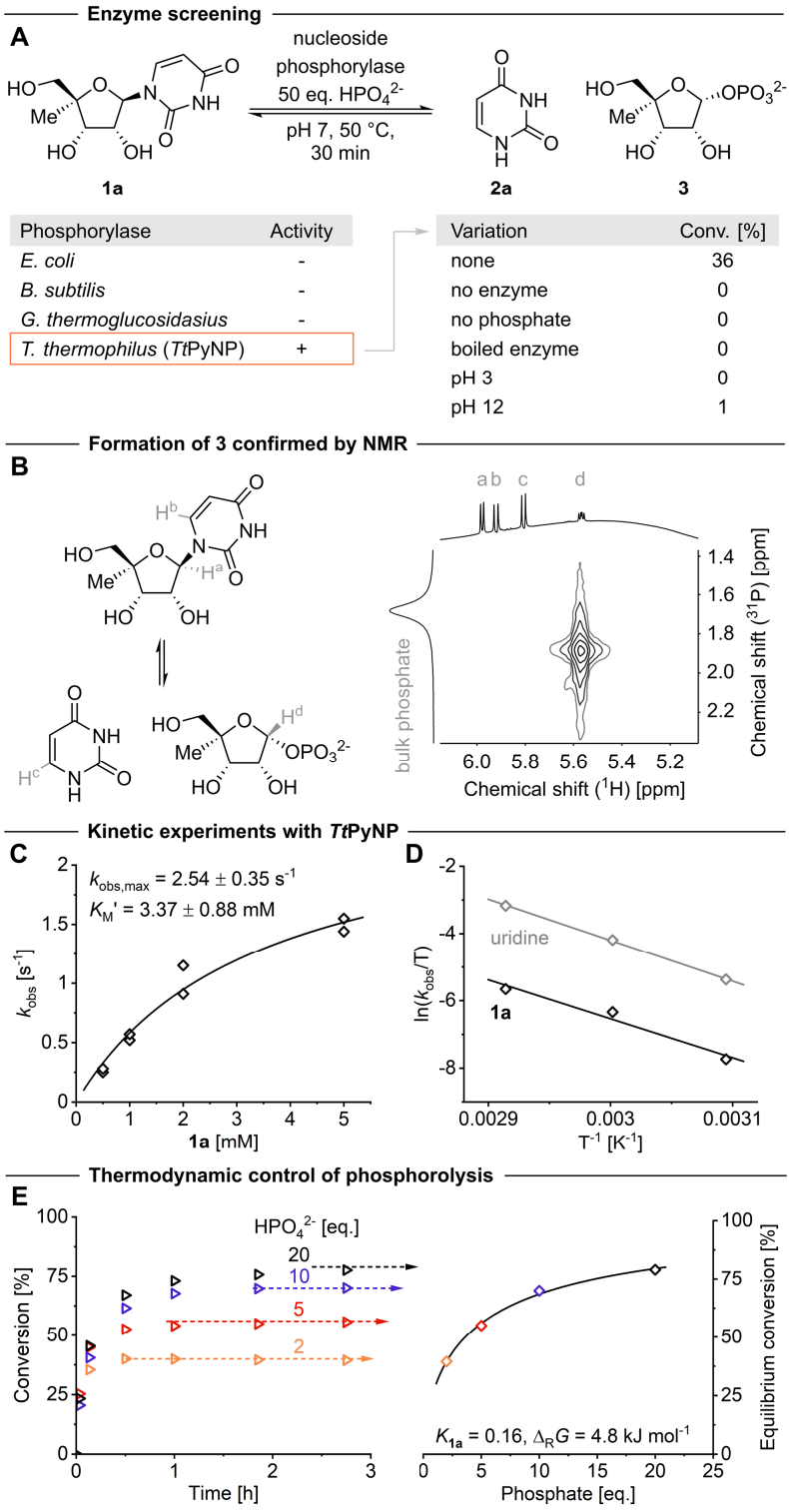

Figure 1. Phosphorolysis of 4'-methyluridine (1a). The data for uridine in D were taken from ref. 26. Please see the Supporting Information for details and the externally hosted supplementary information for raw data. ${ }^{[33]}$

transformation, as evident from the order of magnitude difference between the rate constants of the active enzymes, but these must arise from mutations far from the active site, as all other residues in possible contact with the substrate are identical between the tested enzymes.

Since the phosphorolysis of ribosyl and 2'-desoxyribosyl nucleosides is under tight thermodynamic control, ${ }^{[23]}$ we were then interested in the thermodynamics and reversibility of the phosphorolysis of 1 a to enable a diversification of the scaffold via transglycosylation. Time-course experiments with 1a and varying excesses of phosphate revealed incomplete conversion of the substrate, with the equilibrium positions being consistent with an equilibrium constant $K$ of 0.16 (at $60^{\circ} \mathrm{C}$ and $\mathrm{pH} 9$, Figure 1D). Further experiments to monitor the equilibrium at $75^{\circ} \mathrm{C}$ and $90{ }^{\circ} \mathrm{C}$ revealed that the

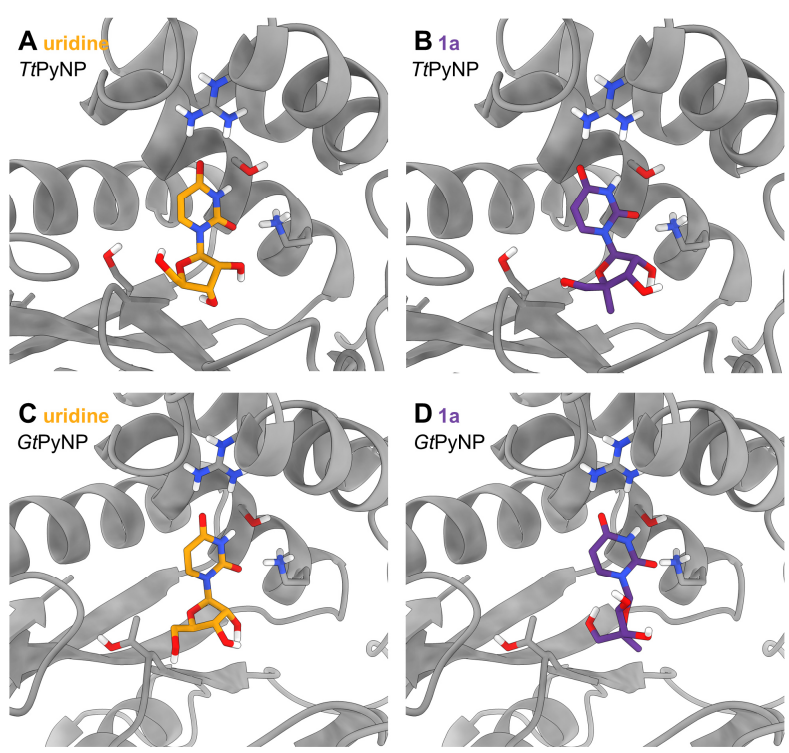

Figure 2. Docking of uridine and 1a into the crystal structures of $T t P y N P$ (A and B) and GtPyNP (C and D) points to a key serine/threonine substitution.

phosphorolysis of 1a has an apparent reaction enthalpy $\Delta_{R} H^{\prime}$ of $8.9 \mathrm{~kJ} \mathrm{~mol}^{-1}$ and an apparent reaction entropy $\Delta_{R} S^{\prime}$ of $11.7 \mathrm{~J} \mathrm{~mol}^{-1} \mathrm{~K}^{-1}$ (Figure S2). Interestingly, these values closely resemble the equilibrium constants and thermodynamic parameters of the phosphorolysis of uridine, ${ }^{[23]}$ indicating that substitutions distant from the anomeric center have little influence on the equilibrium thermodynamics of nucleoside phosphorolysis. These results also pointed to the reversibility of this transformation, opening the door for transglycosylation reactions from the sugar phosphate $\mathbf{3}$ to yield other nucleosides.

With a solid understanding of the thermodynamics and kinetics of the phosphorolysis of $1 \mathrm{a}$ by $T t \mathrm{PyNP}$, we proceeded to diversify this scaffold by subjecting the sugar phosphate 3 to subsequent enzymatic catalysis with different nucleobases in situ. Using this transglycosylation approach (Figure 3A and Scheme 1, center), we aimed to access a variety of $4^{\prime}$-methylated nucleosides from $\mathbf{1 a}$ in a one-pot manner. After confirming the stability of $\mathbf{3}$ through equilibrium shift experiments (Figure S6) ${ }^{[35]}$ we subjected 1a to phosphorolysis using only minimal phosphate in the presence of different pyrimidine nucleobases $\mathbf{2 b}-\mathbf{2 e}$ belonging to a panel of 5-substituted uracil analogs (Figures $3 \mathrm{~A}$ and $3 \mathrm{~B}$ ). Analysis of the reaction mixtures by HPLC revealed consumption of $1 \mathrm{a}$ and the respective uracil analog with concurrent formation of new products (Figure 3B), which HRMS analysis identified as the nucleoside products arising from glycosylation of $\mathbf{2} \mathbf{b}-\mathbf{2 e}$ with $\mathbf{3}$. Equilibrium state thermodynamic calculations ${ }^{[24]}$ based on transglycosylation experiments with different sugar donor concentrations revealed apparent equilibrium constants of phosphorolysis of $0.12-0.73$ for these products $\mathbf{1 b}-\mathbf{1 e}$ (Figure 3B and S3). The trifluoromethylated pyrimidine $\mathbf{2 f}$ could also be converted, although the instability of the starting material and product in aqueous solution ${ }^{[36]}$ 


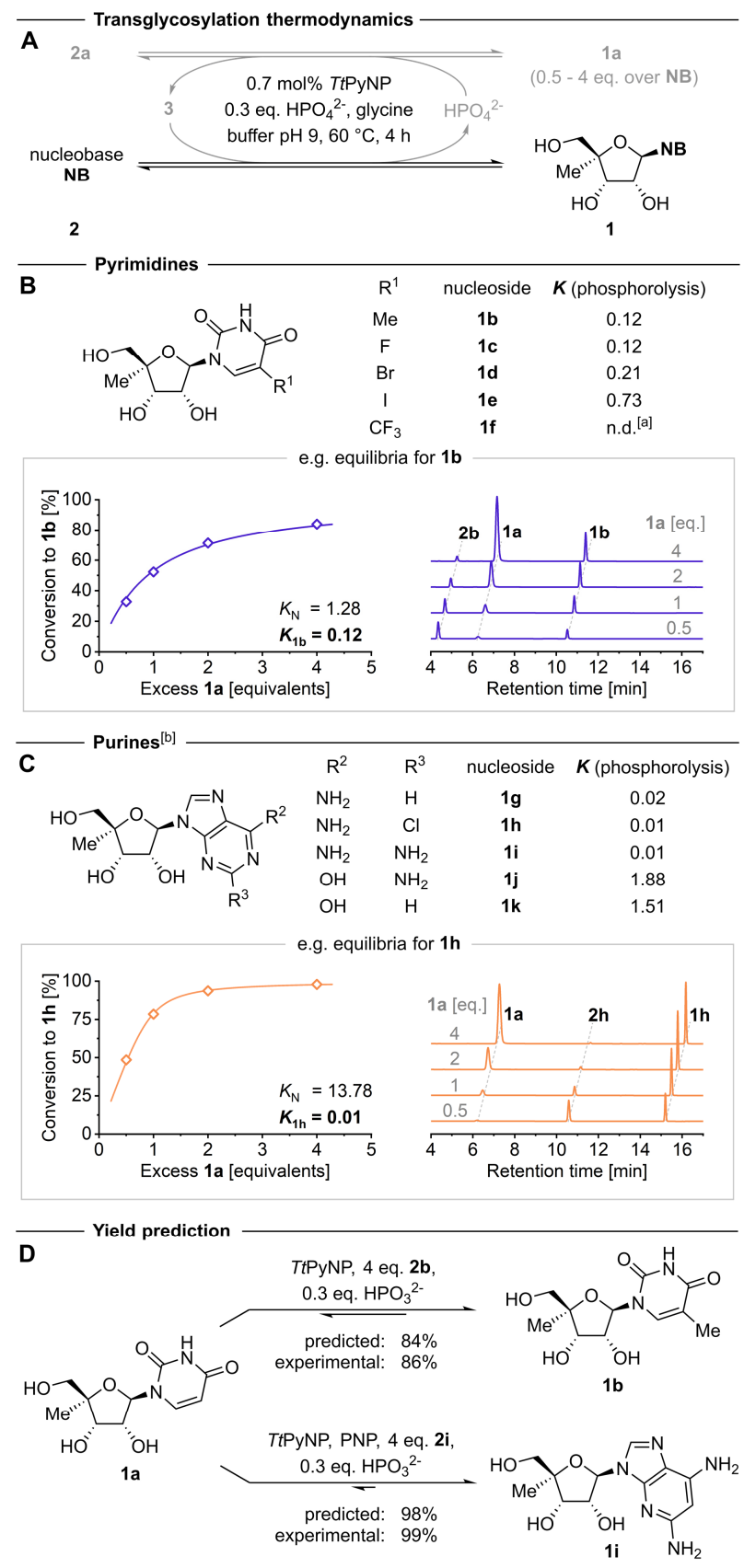

Figure 3. Transglycosylation reactions (A) provided access to different pyrimidine $(\mathbf{B})$ and purine nucleosides $(\mathbf{C})$ as well as the equilibrium thermodynamics of the process, which can be exploited for yield prediction (D). [a] $\mathbf{2 f}$ is converted, but $\mathbf{1 f}$ and $\mathbf{2 f}$ hydrolyse to the corresponding carboxylates under the reaction conditions. [b] Reaction mixtures with purines additionally contained the purine nucleoside phosphorylase from Geobacillus thermoglucosidasius (PNP). Please see the externally hosted supplementary information for raw data and calculations. ${ }^{[33]}$

precluded us from obtaining equilibrium data (Figure S4). A similar elaboration of in situ generated $\mathbf{3}$ with purine nucleobases proceeded smoothly using the promiscuous purine nucleoside phosphorylase from Geobacillus thermoglucosidasius. ${ }^{[37]}$ Notably, the adenosine analogs 1g-1i were generated in much higher conversions, corresponding to equilibrium constants of phosphorolysis of $0.01-0.02$, reflecting the more favorable thermodynamics typically observed for 6-aminopurines. ${ }^{[35,38-40]}$ The guanosine and inosine analogs $\mathbf{1} \mathbf{j}$ and $\mathbf{1 k}$ could also be accessed, although with lower conversions indicative of higher equilibrium constants (Figure 3C). These experiments not only confirmed that nucleoside transglycosylations with the methylated precursor 1a can deliver a range of modified nucleosides in a one-pot manner, but also that the equilibrium thermodynamics of this system largely resemble those of the well-described ribosyl nucleosides. These findings further indicated that these transglycosylations would offer themselves to rational reaction engineering using established principles of thermodynamic reaction control to predict and maximize conversions in these reactions. ${ }^{[24]}$ Indeed, thermodynamic calculations based on the obtained equilibrium constants suggested that $\mathbf{1 b}$ could, for instance, be obtained in $84 \%$ conversion from 1a using 4 equivalents of nucleobase, which we confirmed experimentally (Figures 3D and S5). Similarly, 1i could be obtained in quantitative conversion with 4 equivalents of $\mathbf{2 i}$, in agreement with our predictions. As a proof of synthetic utility, we subjected 1a to transglycosylation with 5 equivalents of $2 \mathbf{e}$ and obtained the iodinated $1 \mathrm{e}$ in $68 \%$ conversion $(61 \%$ predicted) and ca. $40 \%$ isolated yield.

In conclusion, we identified and characterized $T t$ PyNP as a biocatalyst for the diversification of $4^{\prime}$ methylated nucleosides. Reversible phosphorolysis of a methylated precursor 1a yields stable the pentose-1phosphate $\mathbf{3}$ which can be employed as a sugar synthon to access a range of modified nucleosides in one pot. Our investigations revealed that sufficient space near the active site in the open conformation of PyNPs appears crucial for binding and conversion of 1a. Furthermore, the equilibrium thermodynamics of the phosphorolysis of 4'-methylated nucleosides largely resemble those of ribosyl nucleosides, indicating that substitutions distant from the anomeric position have only minor effects on the conversions in these systems. Leveraging principles of thermodynamic reaction control enabled us to access a spectrum of 4'-methylated nucleosides bearing different pyrimidine and purine bases in transglycosylation reactions. Lastly, we expect that other 4'-modified nucleoside analogs can be obtained with such biocatalytic systems in a similar fashion (probably with comparable equilibrium thermodynamics), although bulkier 4 '-substitutions will likely require some extent of protein engineering to improve activity.

\section{Acknowledgements}

The authors thank Kerstin Heinecke (Technische Universität Berlin) and Dr. M. Rhia L. Stone (Rutgers University) for proofreading and critical comments. The authors further thank the Analytical Department of the Technische Universität Berlin for HRMS analyses - Dr. Schlangen und Mr. Griffel do an amazing job. P.P. was a recipient of a fellowship of the International Max-PlanckResearch School for Environmental, Cellular, and Molecular Microbiology (IMPRS-Mic). G.B. thanks the LOEWE excellence initiative for financial support. P.P. and G.B. acknowledge the excellent support by the European 
Synchrotron Radiation Facility (ESRF), Grenoble, France. We thank Laura Czech for correspondence on the experimental details.

\section{Author Information}

Corresponding author

Felix Kaspar, felix.kaspar@web.de, orcid.org/0000-0001-6391-043X

\section{Other authors}

Margarita Seeger, orcid.org/0000-0002-5609-6844

Sarah Westarp, orcid.org/0000-0001-6498-8810

Christoph Köllmann

Anna P. Lehmann, orcid.org/0000-0002-9018-1014

Patrick Pausch, orcid.org/0000-0001-8947-9107

Sebastian Kemper, orcid.org/0000-0003-0192-518X

Peter Neubauer, orcid.org/0000-0002-1214-9713

Gert Bange, orcid.org/0000-0002-7826-0932

Anett Schallmey, orcid.org/0000-0002-6670-0574

Daniel B. Werz, orcid.org/0000-0002-3973-2212

Anke Kurreck, orcid.org/0000-0001-6919-725X

\section{References}

[1] G. M. Blackburn, M. J. Gait, D. Loakes, D. M. Williams, Eds. , Nucleic Acids in Chemistry and Biology, Royal Society of Chemistry Publishing, 2006.

[2] L. P. Jordheim, D. Durantel, F. Zoulim, C Dumontet, Nat. Rev. Drug Discov. 2013, 12, 447464.

[3] J. Shelton, X. Lu, J. A. Hollenbaugh, J. H. Cho, F. Amblard, R. F. Schinazi, Chem. Rev. 2016, 116, 14379-14455.

[4] E. De Clercq, Rev. Med. Virol. 2009, 19, 287-299.

[5] W. Xu, K. M. Chan, E. T. Kool, Nat. Chem. 2017, 9, 1043-1055.

[6] C. Y. Jao, A. Salic, Proc. Natl. Acad. Sci. 2008, 105, 15779-15784.

[7] F. Kaspar, M. R. L. Stone, P. Neubauer, A. Kurreck, Green Chem. 2020, DOI 10.1039/D0GC02665D.

[8] P. Merino, Ed. , Chemical Synthesis of Nucleoside Analogues, Wiley, 2013.

[9] S. Kamel, H. Yehia, P. Neubauer, A. Wagner, in Enzym. Chem. Synth. Nucleic Acid Deriv., 2019, pp. 1-28.

[10] H. Vorbrüggen, C. Ruh-Pohlenz, Org. React. 2004, $1-630$.

[11] C. Köllmann, S. M. Sake, P. G. Jones, T. Pietschmann, D. B. Werz, J. Org. Chem. 2020, 85, 4267-4278.

[12] C. Köllmann, S. M. Wiechert, P. G. Jones, T. Pietschmann, D. B. Werz, Org. Lett. 2019, 21, 6966-6971.

[13] G. Wang, N. Dyatkina, M. Prhavc, C. Williams, V. Serebryany, Y. Hu, Y. Huang, J. Wan, X. Wu, J. Deval, et al., J. Med. Chem. 2019, 62, 4555-4570.

[14] L. Eyer, R. Nencka, I. Huvarová, M. Palus, M. Joao Alves, E. A. Gould, E. De Clercq, D. Růžek, J. Infect. Dis. 2016, 214, 707-711.

[15] K. Vejlegaard, C. Wegeberg, V. McKee, J. Wengel, Org. Biomol. Chem. 2018, 16, 13121321.
[16] P. Franchetti, L. Cappellacci, M. Pasqualini, R. Petrelli, P. Vita, H. N. Jayaram, Z. Horvath, T. Szekeres, M. Grifantini, J. Med. Chem. 2005, 48, 4983-4989.

[17] H. Ohrui, Chem. Rec. 2006, 6, 133-143.

[18] M. K. Yates, K. L. Seley-Radtke, Antiviral Res. 2019, 162, 5-21.

[19] M. McLaughlin, J. Kong, K. M. Belyk, B. Chen, A. W. Gibson, S. P. Keen, D. R. Lieberman, E. M. Milczek, J. C. Moore, D. Murray, et al., Org. Lett. 2017, 19, 926-929.

[20] M. Brodszki, B. Bäckström, K. Horvath, T. Larsson, H. Malmgren, M. Pelcman, H. Wähling, H. Wallberg, J. Wennerberg, Org. Process Res. Dev. 2011, 15, 1027-1032.

[21] M. Meanwell, S. M. Silverman, J. Lehmann, B. Adluri, Y. Wang, R. Cohen, L.-C. Campeau, R. Britton, Science 2020, 369, 725-730.

[22] P. N. Edwards, J. Enzyme Inhib. Med. Chem. 2006, 21, 483-518.

[23] F. Kaspar, R. T. Giessmann, P. Neubauer, A. Wagner, M. Gimpel, Adv. Synth. Catal. 2020, 362, 867-876.

[24] F. Kaspar, R. T. Giessmann, K. F. Hellendahl, P. Neubauer, A. Wagner, M. Gimpel, ChemBioChem 2020, 21, 1428-1432.

[25] M. A. Huffman, A. Fryszkowska, O. Alvizo, M. Borra-Garske, K. R. Campos, K. A. Canada, P. N. Devine, D. Duan, J. H. Forstater, S. T. Grosser, et al., Science 2019, 366, 1255-1259.

[26] F. Kaspar, P. Neubauer, A. Kurreck, ChemBioChem 2020, $n / a$, DOI 10.1002/cbic. 202000679 .

[27] M. Almendros, J. Berenguer, J.-V. Sinisterra, Appl. Environ. Microbiol. 2012, 78, 3128-3135.

[28] K. Szeker, X. Zhou, T. Schwab, A. Casanueva, D. Cowan, I. A. Mikhailopulo, P. Neubauer, J. Mol. Catal. B Enzym. 2012, 84, 27-34.

[29] F. Kaspar, R. T. Giessmann, N. Krausch, P. Neubauer, A. Wagner, M. Gimpel, Methods Protoc. 2019, 2, 60.

[30] F. Kaspar, R. T. Giessmann, S. Westarp, K. F. Hellendahl, N. Krausch, I. Thiele, M. C. Walczak, P. Neubauer, A. Wagner, ChemBioChem 2020, 21, 2604.

[31] M. J. Pugmire, S. E. Ealick, Structure 1998, 6, 1467-1479.

[32] K. Shimizu, N. Kunishima, 2006, DOI 10.2210/pdb2DSJ/pdb.

[33] F. Kaspar, 2021, DOI 10.5281/zenodo.4630205.

[34] X. Ren, N. Liu, A. L. Chandgude, R. Fasan, Angew. Chem. Int. Ed. 2020, 59, 21634-21639.

[35] F. Kaspar, P. Neubauer, A. Kurreck, ChemPhysChem 2020, $n / a$, DOI 10.1002/cphc.202000901.

[36] C. Heidelberger, D. G. Parsons, D. C. Remy, J. Med. Chem. 1964, 7, 1-5.

[37] X. Zhou, K. Szeker, B. Janocha, T. Böhme, D. Albrecht, I. A. Mikhailopulo, P. Neubauer, FEBS J. 2013, 280, 1475-1490.

[38] H. Yehia, S. Westarp, V. Röhrs, F. Kaspar, T. R. Giessmann, F. T. H. Klare, K. Paulick, P. Neubauer, J. Kurreck, A. Wagner, Molecules 2020, 
25, 934 .

[39] C. S. Alexeev, I. V Kulikova, S. Gavryushov, V. I. Tararov, S. N. Mikhailov, Adv. Synth. Catal. 2018, 360, 3090-3096.

[40] R. A. Alberty, J. Chem. Thermodyn. 2004, 36, 593-601. 